\title{
LOS TALLERES DE EDUCACIÓN DE LA SEXUALIDAD Y LA AFECTIVIDAD EN LOS ADOLESCENTES COMO UN MEDIO DE PREVENCIÓN DELABUSO SEXUAL (UNA PROPUESTA METODOLÓGICA Y TEÓRICA)
}

\author{
Margarita Murillo Gamboa*
}

Fundamentada en los aportes de Erich Fromm, acerca del tener y del ser, la autora elabora una propuesta para desarrollar un taller orientado a la prevención del abuso sexual en adolescentes. De esta forma, busca que los y las jóvenes se involucren en la actitud de ser y no tanto del tener. Asimismo, se aborda el concepto de sexualidad que los participantes tenían antes y después del taller.

El presente artículo se complementa con el suplemento "Mi sexualidad", escrito por la psicóloga Pamela Delgado, dirigido a estudiantes de cuarto, quinto y sexto grado de la Educación Primaria. Este suplemento complementa la revista que usted tiene en sus manos.
Based on the contributions made by Erich Fromm, in regards to the concept of "having and being," the author proposes the development of a workshop aimed at preventing sexual abuse of adolescents. In this way, she tries to attract youngsters more into the attitude of "being" rather than into that of "having." Likewise, the participants' concept of sexuality before and after the workshop is discussed. This article is completed with the supplement "Mi sexualidad" ("My Sexuality"), written by psychologist Pamela Delgado, and addressed to fourth, fifth and sixth graders. It complements the magazine you are reading.

* Estudiante del Doctorado en Sociedad y Cultura. Máster en Investigación en Psicología, Universidad de Costa Rica. Elabora una propuesta de metodología de abordaje en educación de la sexualidad. Columnista por tres años de los periódicos La Prensa, Nicaragua. Columna "Construyendo la Sexualidad, La Nación, Costa Rica y en periódicos de la provincia de Heredia, Alajuela y Cartago. Colaboradora en la Revista Dominical, Revista Viva, Revista Perfil. Ha escrito ar culos de apoyo en el periódico Reforma- México y El Reportero de San Francisco, California. Está por publicarse su libro El Lenguaje de la sexualidad como una propuesta interactiva de formación en educación de la sexualidad. Es directora de la Revista Costarricense de Psicología. 
Durante estos años de investigación con talleres cuyo tema ha sido la afectividad y la sexualidad y cuya población, en la mayoría de las veces, ha sido con adolescentes se buscó como objetivo principal ofrecer un espacio que los forme, les permita reflexionar, determinar y proporcionar alternativas de vida; sobre todo basados en la posición de que, precisamente en el período de la mediana adolescencia, será donde se comienza a desarrollar la tarea de convertirse en hombres y mujeres $\mathrm{y}$, al mismo tiempo, es un momento dentro de su crecimiento que les permite poder comprender el desarrollo sexual como parte de su crecimiento.

La teoría que dirige, motiva y a la vez enfoca el abordaje en estos talleres es aquella que incentiva la toma de decisiones de tal manera que se estimula en ellos y ellas un espacio de reflexión que los ayude a formarse y que a la vez los y las comprometan con los logros posibles de una civilización más justa y equilibrada para todos (as), con esta posición será indispensable resaltar que el punto de partida de estos talleres es reconocer en el y la adolescente la capacidad de aportar y desarrollarse con responsabilidad propia dentro de su comunidad.

Esta posición teórica es la guía para el manejo de los procesos de reflexión, buscando con este enfoque generar un cambio de actitud y una estimulación en la toma de conciencia, el cual está basado en los aportes de Erich Fromm (1992) de sus libros ¿Ser o tener? Y Del tener hacia el ser, dado que su posición teórica, además de acertada, aclara la perspectiva que se busca en la formación propuesta.

Dentro de sus postulados este autor menciona que en este momento histórico se ha construido una cultura del tener, en donde se vende "la gran promesa" (proyecto de vida) que dice que los logros materiales e intelectuales son los éxitos en la vida del hombre y la mujer y estos logros son los que los y las conforman en su modo de vida. Bajo esta perspectiva, hombres y mujeres hemos venido construyendo y vivenciando la sexualidad y será necesario enseñar el efecto enajenante que se suscita en la vivencia de la sexualidad cuando se vive desde una cultura del tener y no del ser, que es lo que se debe proponer a los jóvenes.

Dos premisas psicológicas de la teoría de E. Fromm (1992) serán los complementos que se deben tomar en cuenta en el abordaje y, al mismo tiempo, permitirá orientar el enfoque con que se estimule el análisis individual y colectivo. Con estos aportes se busca generar cuestionamientos acerca de la vivencia de los postulados en la construcción de lo que se desea ser como personas, estas premisas son las siguientes:

1. Creer que la meta de la vida es la felicidad, entendida la meta como un máximo de placer, que se define como la satisfacción de todo deseo o necesidad subjetiva que una persona pueda tener (hedonismo radical). Es una premisa que debe buscar analizarse y reflexionarse con los y las jóvenes 
como parte del proceso de encuentro y formación. Dado que la sexualidad se asocia como una "fuente de felicidad" y placer hay que aclarar que ella por sí misma no es felicidad ni placer, sino un medio de salud que genera placer. La felicidad es un proceso del desarrollo humano que se construye en la formación y encuentro con el ser no por medio de la sexualidad.

2. El egoísmo y la avaricia que el sistema necesita fomentar para funcionar hace creer a las personas que esta forma de vida (egoísta y avara) es la que produce armonía y paz. Ante esta premisa también se deben generar espacios que desarrollen una toma de conciencia para lograr identificar y manejar adecuadamente las actitudes que se generan con la vivencia de la sexualidad, pues se mira a esta como un espacio para satisfacerse a sí mismo (a) y no como una fuente de salud tanto para sí como para el otro y la otra, es lograr entrar dentro de sí para generar placer, pero al mismo tiempo tener la capacidad de salir de sí mismo para poder encontrarme con el otro y la otra.

Con estas premisas se estimula una posición en el facilitador (a) que busque generar conciencia en los y las participantes acerca de la influencia que estos postulados tienen, no solo en la vida cotidiana, sino sobre la forma en que abordamos y vivimos la sexualidad, se debe destacar en el espacio del taller, que las ideas que la sociedad actual estimula, son aquellas que mencionan que hay que librarse de los vínculos del amor y de la solidaridad y que se puede vivir sólo para uno (a) mismo (a) y el resultado final será ser más y no menos esto, simplemente solo genera vacío y ambigüedad.

Estos temas o premisas deben de servir de guía dado que estas actitudes contradictorias corresponden a una necesidad económica del capitalismo del siglo XX que basa sus ideas en el consumo de bienes y el uso de servicios al máximo, así como en el rutinario trabajo de conjunto lo que afecta directamente la vivencia y conceptualización de la sexualidad. Sobre todo al objetivizar la vivencia y desarrollo de esta como un objeto más de consumo y no como fuente creadora y de placer dentro del desarrollo humano.

De hecho la alternativa entre TENER y SER, no atrae al sentido común, parece que TENER es una función normal de la vida, para vivir debemos TENER cosas, además, debemos tenerlas para gozarlas. Es una cultura cuya meta suprema es TENER CADA VEZ MÁS, incluso refiere que la esencia del SER consiste en TENER, y si el individuo NO TIENE NADA, NO ES NADIE.

Ofrecerles a los jóvenes un espacio para tomar conciencia sobre estas posiciones en el taller de sexualidad será estimular un proceso de darle al mismo mundo la conciencia de lo que es. La conciencia es por sí misma la actividad de ordenar el contenido de los sentidos. El tomar conciencia de la influencia del 
Si como hombres y mujeres nos vemos limitados en la comunicación adecuada de nuestras necesidades afectivas, se limitará el desarrollo máximo del potencial orgásmico - erótico y del potencial afectivo - espiritual que será la fortaleza que necesitaremos para ver con más claridad los valores a vivir en la construcción de la relación sexual y del desarrollo sexual en general; lo que queremos, cómo lo queremos y cuándo lo queremos, sin tener que caer en una relación de opresión y violencia.

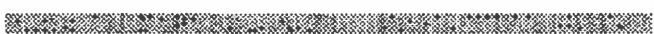

tener sobre el ser podrá estimular la claridad de percibir qué tanto afecta a la humanidad los actos y decisiones personales. Todos somos UNO y es menester de todos (as) cuidarnos y crecer en UNIDAD, que fue algo que perdimos al ser considerados como máquinas o como objetos aislados. Esto lo destaco, pues la actitud al abordar la sexualidad está regida por estos conceptos de una cultura de TENER, por ejemplo, "tener relaciones" "tener novio (a)", es objetivizar una energía de la salud y que complementa al SER no que lo posee. Esto raya en el ámbito del poder que surge en la vivencia de la sexualidad y sobre el que hay que abordar con sumo cuidado y profundidad.

En estos talleres se busca promover, en los y las jóvenes, por medio de la reflexión y otros ejes ${ }^{1}$, espacios que los estimulen a distinguirse dentro de la humanidad. Si no se realiza esta reflexión no se podría generar en ellos una reconceptualización de la vivencia de la moralidad y la plenitud y certeza de reconocer en su sexualidad una energía de vida y no de muerte.

Uno de los problemas que se tienen al investigar en este campo es la percepción limitada de la realidad y, con esta limitación, pretender abordarla sin considerar las necesidades socioculturales e históricas de los diferentes grupos humanos. En ocasiones estas limitaciones se convierten en "facturas" (obligaciones) que llegan a obstaculizar la concepción y los objetivos de la afectividad y la sexualidad y, por ende, el abordaje que se realiza con los y las adolescentes.

Este artículo busca ser un complemento teórico orientador al folleto de formación en Afectividad y Sexualidad elaborado por la Licda. Pamela Delgado, ella buscó sistematizar una propuesta educativa basada en estos postulados, en los ejes metodológicos y en la teoría del sexoanálisis del Modelo de Intervención Global en Sexología de la Dra. M.P. Ross (1998) que puede ser ejecutada con estudiantes de cuarto, quinto y sexto grado de tal manera que quien lo administre en sus grupos educativos tome en consideración estos aspectos.

1 Los ejes metodológicos de los talleres y su aplicación fueron expuestos en el Congreso Nacional de Psicología del 2002, Costa Rica; y pueden ser encontrados en la memoria del Congreso. Así como en el Congreso Nacional de Sexología en abril del 2004, Costa Rica. 
Los referentes teóricos de la teoría del sexoanálisis que aporta la Dra. Marie Paul Ross de la Universidad de Québec, lo que ella denomina "Modelo Global de Intervención en Sexología Clínica", ha sido una corriente teórica basada en los aportes de Claude Crepaùlt y otros investigadores de la Universidad de Québec, Canadá.

Estos aportes vienen a ser un complemento importante en la forma en que se visualiza tanto el desarrollo y vivencia de la sexualidad, así como el compromiso personal que se debe promover y adquirir para desarrollarse a plenitud como hombres y mujeres.

Este enfoque teórico de intervención, que basa sus aportes en la premisa de que la sexualidad está estructurada por componentes, que forman una interrelación y una interdependencia entre ellos. Estos componentes, independientemente, son esenciales para el desarrollo integral de la sexualidad, pero a su vez su desarrollo va a depender de la resolución y equilibrio de los otros dos.

En este enfoque se destacan los siguientes elementos para cada uno de los componentes:

El componente espiritual: se define por aspectos cognoscitivos como el conocimiento, el desarrollo de la comprensión, el aprendizaje y educación que tengamos acerca del desarrolío sexual, la formación, la capacidad de análisis, la toma de decisiones, las elecciones que se toman, el compromiso y la responsabilidad ante mi desarrollo sexual. También tiene un enfoque de visión trascendental, en donde entran factores alrededor de mis creencias, mi sentido de vida y mi sentido de muerte, la conciencia de finitud e inmortalidad, así como la construcción que realice acerca del amor perpetuo y la vida perpetua con mis decisiones. Tiene también un sentido espiritual (no religioso $)^{2}$ cuando se toma conciencia de las construcciones y decisiones acerca del perdón, la gratitud, sentir la sexualidad como un don de la vida, poder estructurar adecuadamente el sentido del sufrimiento y del dolor, así como los conceptos de fraternidad, afinidad espiritual y honradez.

Este componente logrará la plenitud de su seguridad como ente indispensable en el desarrollo integral de la sexualidad por medio de la seguridad en la vida y el amor perpetuos y agregaría la conciencia de sentirse dignificados con el propio comportamiento y decisiones que afectan el desarrollo integral de la sexualidad. El poder construir la sexualidad propia, partiendo de que se es un ser digno, único y valioso (a).

2 Se entenderá espiritualidad desde los aportes de Víctor Frank (1994) y su posición dada en el libro El hombre en busca de sentido. Una persona sin prácticas o creencias religiosas de igual manera debe abordar el componente espiritual de la sexualidad. 
El componente erótico: está determinado por factores fisiológicos, genitales, aspectos del desarrollo y manejo de la sensualidad, la belleza corporal, la excitación, los reflejos, la relajación, el manejo de la relación fusional ${ }^{3}$, el placer, la reproducción, la intimidad corporal, el enamoramiento, la pasión amorosa, los deseos sexuales, la experiencia emocional los fantasmas románticos. En este componente la persona logrará la plenitud y seguridad de su desarrollo en la medida en que se pueda establecer una adecuada intimidad corporal y la estructuración de una relación placentera.

El componente afectivo: se va a definir por los elementos que son esenciales al amor, tales como los valores de verdad, respeto, fidelidad, libertad y a partir de mi experiencia terapéutica he podido rescatar aspectos como el diálogo, la responsabilidad y el desarrollo de procesos de aceptación. Se mencionan dos tipos de experiencias en el desarrollo de este componente, como el poder tener un sentimiento de amor con uno mismo, con los demás y con Dios.

También se destaca el poder desarrollar un crecimiento afectivo que tiene que ver con la posibilidad de diferenciarnos, identificarnos, actualizarnos y desarrollar la autonomía afectiva. Además se destacan aspectos como la afinidad afectiva, la amistad y la confianza.

Este componente tiene la variante de ser el eje de equilibrio en el desarrollo adecuado de los componentes eróticos y espirituales, es decir, el componente espiritual necesitará de los elementos del erótico para su adecuado desarrollo y viceversa, pero será por medio de la afectividad, el buen manejo, conocimiento y madurez de esta la que permitirá integrar los elementos necesarios de acuerdo a la etapa del desarrollo en la que nos encontremos.

No es un secreto que muchas de las consultas en sexualidad giran más entorno al tema del manejo de la afectividad en la relación entre hombres y mujeres, así como en la estructuración de lo que se desea o no para la vida personal. Como lo hemos podido observar, este manejo de la afectividad se manifiesta y comunica de manera diferente en hombres como en mujeres, básicamente por el efecto de los patrones culturales (género) que se adquieren cuando se conforman nuestros comportamientos en relación con los roles que se han inculcado.

El componente afectivo es el eje medular de toda relación humana y del desarrollo sexual, por ende, la forma en como se expresen nuesuras emociones, así como la claridad que se tenga hacia la intensidad de estas emociones, hará que esta fuerza de vida-creativa, que es la sexualidad, engendre frutos o no, de hecho, para que el

3 Los aportes de la relación fusional parten de los aportes del psicoanálisis y de la posición teórica de Cavanagh (citado en De Mezerville, G. , 1999) en lo que se refiere a la construcción y componentes de la identidad psicosexual. 
componente erótico pueda lograr una máxima expresión orgásmica estará determinado por la clara conciencia y manejo adecuado de los sentimientos.

Cuando se mencionan los sentimientos, vale destacar que para los hombres y las mujeres estos van a tener formas diferentes de expresión. Analicemos, por ejemplo, lo que menciona la terapeuta Fina Sanz (1999) de España, en donde destaca que para que las mujeres puedan comunicar claramente sus sentimientos deben comenzar a desarrollar un mayor respeto por su palabra, la mujer por lo general, menciona la autora, va utilizar patrones de comunicación, utilizando su cuerpo y no tanto con su palabra. Menciona que tanto el cuerpo como la palabra están dotados de un gran contenido emocional, así como a partir del silencio que tiene un gran trasfondo afectivo de rebeldía y enojo.

Uno de los problemas sociales a los que se enfrentan las mujeres tiene que ver con el valor y peso que se le da socialmente a la palabra de la mujer, lo que diga, tiene socialmente poco valor, se le rechaza o se le cataloga como algo que pierde mucha fuerza porque usa "muchas palabras o habla mucha paja", como si las mujeres no merecieran ser escuchadas y esto afecta profundamente su autoestima y la manifestación clara de su sexualidad que será por medio de palabras y palabras de afecto, de hecho la mujer, al no tener la posibilidad de poder comunicarse clara y abiertamente por medio de una palabra que le sea creíble, podrá llegar a utilizar su cuerpo como vehículo de sus emociones e incluso, de acuerdo a los aportes de esta autora, culturalmente se señala a la mujer de histérica, provocadora o degenerada.

Por el contrario el hombre ha sido educado para manifestar su afectividad por medio de la agresividad, la competitividad, el manejo y dominio del cuerpo de la mujer, llegando las mujeres a establecer una queja generalizada de que los hombres manifiestan su afectividad en caricias que se limitan a los labios, pechos y genitales femeninos.

Josefina Sanz (1999), establece que el hombre limita su expresión a los genitales, la dominación y la expresión hacia afuera, de hecho su palabra es palabra de poder, es la que se escucha, tenderá a utilizar más los conceptos abstractos, preferirá hablar de lo ajeno a los propios sentimientos y tendrá poco control de su cuerpo o de la expresión de su excitación.

Si como hombres y mujeres nos vemos limitados en la comunicación adecuada de nuestras necesidades afectivas, se limitará el desarrollo máximo del potencial orgásmico-erótico y del potencial afectivo-espiritual que será la fortaleza que necesitamos pàra ver con más claridad los valores a vivir en la construcción de la relación sexual y del desarrollo sexual en general; lo que queremos, cómo lo queremos y cuándo lo queremos, sin tener que caer en una relación de opresión o violencia. 
Todo esto es una forma de denunciar el maltrato que se da en nuestras relaciones diarias de convivencia, se procura fomentar que tanto hombres como mujeres abran o aprendan a establecer espacios para conversar, eso implica hablar y escuchar, aunque eso culturalmente sea "muy femenino", hará que cualquier relación crezca profundamente, y por otro lado, las mujeres deberían fortalecerse en seguir luchando por hacer valer su palabra, por llegar a acuerdos, y no limitarse a callar y"dejar pasar las cosas", porque las cosas no pasan solo se acumulan, y son como una olla de presión, en cualquier momento explotan y todo salta y golpea por todos lados. Este es una posición que será determinante desarrollar en los talleres con los y las adolescentes, de ahí la necesidad de estimular la reflexión y el diálogo.

Así que para poder desarrollar el componente afectivo, que es el equilibrio de nuestra sexualidad, es imprescindible que los varones aprendan a comunicar sus emociones y aprendan a comunicarse, conocer y controlar su cuerpo, y por el otro, las mujeres hagan valer su palabra y puedan manifestar abiertamente sus necesidades de darse un espacio para disfrutar de la sexualidad como algo sano y natural dentro del desarrollo humano.

También, vale la pena rescatar los procesos de concientización que se han venido estructurando en diferentes círculos sociales de profesionales y de personas no profesionales, acerca del significado del placer, por lo general se nos ha vendido la idea barata de que el placer dependerá del dominio que tengamos a nivel teórico-práctico de posiciones o puntos orgásmicos, que han venido a nuestras mentes más por influencia comercial, en donde se mencionan actividades que por la fantasía con que las identifican nos hacen creer que se logrará una mejor vivencia del placer, sin "preocupaciones" o sin tomar en cuenta nuestros sentimientos y los (as) de los otros (as), nuestras necesidades, es simplemente creer que el placer por sí mismo reacciona y eso no es real. A menudo en la terapia clínica se logra observar en los (las) pacientes la forma como van perdiendo la capacidad de sentir placer por el simple hecho de no apreciarse a sí mismos, no se puede incluso confundir placer con eyaculación, el placer por sí mismo es un elemento del componente erótico, pero el orgasmo es una expresión erótica, espiritual y afectiva, y para lograrlo se requiere del equilibrio y la manifestación y realización de todos ellos.

Para lograrlo se debe desacomodar de la mente esos actos mecánicos que nos venden de forma precaria en cuanto video musical observamos, o en películas pornográficas cargadas de violencia contra la mujer y la vivencia del placer de ambos. Solo son desviadoras de la verdadera dimensión del placer, se ha promocionado una sexualidad completamente ajena de nosotros mismos, de lo que creemos o no creemos, incluso, por patrones culturales a las mujeres se les 
corta la manifestación abierta de su placer o deseo, pues culturalmente eso es "mal visto en una mujer decente", y por esto las mujeres llegan a perder el potencial y el desarrollo de su madurez sexual.

El componente erótico también se desarrolla por medio de lo fisiológico, el uso y aceptación del cuerpo, la genitalidad, y esto implica el conocimiento de las manifestaciones de la sensualidad en nuestro cuerpo e incluso también por medio de nuestros genitales (esto implica que no es solo por medio de los genitales), además, es de gran ayuda cuando ya se ha adquirido la madurez de una relación que conlleva compromiso, espacio, tiempo, construcción de realización en los ámbitos de la autonomía y seguridad en lo que se desea ser como hombres y mujeres libres. Requiere de un diálogo profundo, conmigo mismo (a), y con el otro (a).

El factor erótico viene a ser un proceso de mirar la belleza en aquellos momentos de frustración en las noches de sacar cólicos a los (as) hijos (as), con una verdadera "cara de locos y trasnochados (as)" sin parranda incluida. Es lograr mirarse bellos en esos espacios de juegos, esto se debe fomentar en los y las adolescentes como nuevos espacios por conocerse tanto a a sí mismos (as) como en relación con los pares. Es estimular que busquen determinar valores, sueños o construcción de la autonomía de ambos.

La sexualidad merece mucho respeto como para tomarlo a la ligera, no es una excitación barata, no son solo reflejos para encontrar los puntos orgásmicos, o un proceso de aprender a relajarnos, implica construir una verdadera relación fusional en donde el placer, la reproducción, y no solo la reproducción de posiciones u orgasmos, sino estimular la reproducción de sus metas a nivel personal, profesional y social. Es esa intimidad corporal, de apreciarse y amar, es construir un verdadero enamoramiento que perdure, es la pasión amorosa y los deseos sexuales manifestados por medio de la experiencia emocional y los fantasmas románticos.

Es buscar la seguridad y desarrollo de ti y de mí por medio de la intimidad corporal y la emoción placentera.

\section{Características del taller}

Lo que se propone es estructurar talleres en donde los y las estudiantes apliquen lo antes expuesto y puedan desarrollarse espacios de crecimiento y compromiso por parte de ambos, así como de sus maestros (as) y padres y madres. También como lo que ofrece la Licda. Pamela Delgado con su propuesta pedagógica para estudiantes de cuarto, quinto y sexto grado de educación primaria. 
El taller deberá estructurarse como un espacio para informarse, formarse y reflexionar, para tomar posición, para entrar en uno mismo 4 .

Se busca ofrecer un proceso de aprendizaje bajo una perspectiva de aprehender desde el modo de SER, esto implica un compromiso en el reconocimiento, primero, de sus sentimientos y luego de la relación que estos guardan con sus necesidades humanas, en vez de que los y las adolescentes sean recipientes pasivos de las palabras y de las ideas, busca que ellos y ellas escuchen, capten y respondan de una manera productiva y activa.

Mucho se ha hablado de crisis, y de crisis sexual, de violencia y abusos, mucho de esta realidad se le mira desde fuera de nosotros, fuera de nuestra cotidianidad, como si no tuviera relación con las decisiones, como si esta crisis de la que se habla no tuviera repercusiones sobre nuestra forma de interrelacionarnos. Ante esta actitud se evidencia una posición desde la visión del TENER, es una actitud hostil y egoísta. Se ha creado, por tanto, un temor a SER.

Basados en esta necesidad de fortalecer el desarrollo del ser en los y las adolescentes es que se estructuran y se construyen estos talleres. Se busca que de los y las participantes encuentren sus habilidades y sus debilidades, que tome conciencia de esto y pueda ser asumido como un proyecto de vida.

Se define, conceptualmente, de que el abuso sexual forma parte del inadecuado manejo del deseo, del placer, del poder y de las relaciones interpersonales, así como del desarrollo integral del ser. El abuso sexual es una muestra más de la inequidad y explotación que se vive en el desarrollo desigual de los géneros y de la humanidad en general.

Poder tomar conciencia y fortalecer las bases en la construcción de la identidad psicosexual es una de las tantas formas que se pueden ofrecer como alternativas de desarrollo sexual más justas, equitativas y con un adecuado manejo del placer.

Mientras los jóvenes no se involucren con una actitud de SER, no habrá ningún cambio para la sociedad y se pasará a formar parte de las cosas y los objetos que nos rodean. La evolución no se dará ni se desarrollará a plenitud si no se logra fortalecer y establecer una autodeterminación que viene a ser como el hilo conductor de la evolución.

Ambos, hombres y mujeres, han llegado a perder la direccionalidad y la autodeterminación en relación con su sexualidad. Esta, la sexualidad, ha perdido sus dimensiones conscientes para convertirse en un acto trivial, en donde no existe o no

4 Las propuestas de taller y temas serán expuestos en otro artículo, también se puede consultar la página web: www.sexoamoryvida.com para referencias. En este artículo se recomiendan visiones teóricas sobre la forma en que se deben abordar independientemente de la temática. 
se estimula la autoorganización ni la autorenovación, ni la autoconservación. La sexualidad ha perdido su relación con el desarrollo integral de las personas.

La sexualidad empezó a formar parte de la cultura del tener, es cuando interesa más hablar de posiciones, del número de veces que se "hace el amor" (como si el amor se hiciera) y poco a poco se le desvincula con las emociones y el proceso de evolución del hombre, solo busca el placer propio y no trasciende a las relaciones interpersonales y al desarrollo integral y pleno de la personalidad.

Si se parte de que la conciencia es por sí misma la actividad de ordenar el contenido de los sentidos, tal parece que la sexualidad se vive sin conciencia, no es algo sobre lo que se pueda tener control, es como espontáneo, "instintivo", por un lado es un eje medular de la conducta humana, sin embargo, no se logra comprender y desarrollar con claridad dada la desvinculación y el manejo adecuado que se mantiene con los sentidos y las emociones.

Una sexualidad vista desde la cultura del tener, se limitará a reproducir sensaciones, movimientos y actos, se cobrará por ella, como se cobra por un carro, y se le mutilará en la expresión auténtica de lo que significa para el ser. Comenzará a engendrar enfermedad y muerte. Y al determinarla así, se convertirá en la autodestrucción del género humano.

Dentro de este artículo es interesante mostrar los resultados de la investigación realizada por la autora, en donde bajo la perspectiva de un proceso de taller (M. Murillo, 2001), el cual se vivió con veintiséis jóvenes, trece hombres y trece mujeres, de los cuales el 34\% tenían dieciséis años y el 65,4\% quince, se les administraron cinco sesiones con los principios teóricos y metodológicos antes expuestos, observar la forma en que se enriquecen las respuestas ante la pregunta: para mí la sexualidad es. Esta pregunta se formuló antes de recibirlas cinco sesiones, después de las cinco sesiones se compararon las respuestas para destacar el efecto de abordar a los y las jóvenes bajo estos principios.

Resultados del cuestionario abierto antes y después de las cinco sesiones:

\section{Concepto de sexualidad}

\section{Antes del taller de sexualidad}

- Relaciones entre dos personas sin importar el sexo (61.5\%)

- Relación entre dos personas de distinto sexo (15.4\%).

- Actitud de madurez (3.8\%)

- Educación acerca del sexo (3.8\%)

- Todo lo que nos rodea (3.8 \%)

- El desarrollo de mi ser (7.7\%)

- No responde $(7.7 \%)$ 
- Relaciones de cualquier tipo hombre y mujer.

- Todo lo que nos identifica y rodea en las relaciones con otras personas.

- Es como uno se comporta con los demás y con las relaciones con los demás.

- El conocimiento del yo mismo y los demás.

- Las relaciones entre todos nosotros y el comprender nuestro cuerpo y nuestra forma de ser y pensar.

- Convivir con diferentes personas, compartir situaciones y actividades.

- Una relación entre hombre y mujer, sea moral, psicológica, física, etc.

- Lo que cada persona tiene.

- Relación que hay entre personas.

- Cuando dos personas o varias quieren estar juntas.

- Todo tipo de contacto con el otro sexo.

- Sexo y sus derivados, es la razón de la vida temprana.

- Todo lo relacionado con el sexo, desde las relaciones entre seres sexuales.

- Cualquier relación entre seres humanos.

- Las relaciones entre personas de diferentes sexos, no necesariamente físicas o de cualquier forma.

- Una gran parte de mi vida. Es mi forma de ser y relacionarme.

- Relacionarse con las demás personas.

- Calidad de hombre o mujer que soy. Mis características que reflejo a los que me rodean. El trato consigo mismo. Emociones y cambio de mi cuerpo.

- Es algo que existe en todos los seres humanos y que interviene en la vida de todos.

- Con la sexualidad las personas aprenden a descubrirse a sí mismas o a degradarse, al igual que aprendemos a sentir.

- Es un sentimiento que lo tenemos siempre.

- Los sentimientos, las emociones, etc., que una persona puede experimentar, la atracción y las relaciones para con los demás.

- La forma de tratarlos y de comportarse, además, de cómo me siento con ellos.

- Todo lo que abarca los sentimientos, pasiones y relaciones del ser humano.

- La relación que existe entre dos individuos, algunas veces es más personal que otras.

- En las más personales los dos individuos comparten más que en la menos personales.

- Cuando alguien comparte con otras personas del sexo opuesto. 
Las estadísticas quedaron de la siguiente manera:

Respuestas entorno a:

- Relaciones $31 \%$

- Es comprender y convivir (19.5\%)

- Conocimiento del yo (34\%)

- Contacto sexo-cuerpo (14.62\%)

Estas conclusiones muestran en parte la visión de lo que los jóvenes tienen acerca de la sexualidad, aquí se hace mención de sexualidad como acto físico, genital, y se hace mucha mención acerca de las relaciones, hablan de conocerse, de los cambios físicos y externos. Poco se ahonda hacia la relación profunda con el entorno, se avocan a una situación que se dá o se dará dentro de su proceso de vida.

Es interesante ver los aportes que estos mismos jóvenes hacen luego de pasar un período de reflexión o al menos de cuestionamiento de lo que habían realizado hasta el momento y esperaban ser como hombres y mujeres en un futuro. En este espacio se les dieron conocimientos, pero estos surgieron de sus reflexiones, tuvieron que estructurarlo, organizarlo, tomar conciencia y reflexionar acerca de lo que estaba sucediendo con sus compañeras y compañeros de clase.

En el segundo grupo de respuestas, después de las cinco sesiones, se mantienen los conceptos de acto genital, de relación con el sexo opuesto como en las anteriores; sin embargo, ya hay aportes de que en la sexualidad se involucra comunicación. Se menciona la identidad de la persona como parte de lo que se es y como parte de lo que es dentro del mundo, se habla de actitudes hacia las otras personas, se menciona la paz, se menciona al ambiente, se busca abrir el corazón a la vida, y hacer referencia de valores, tales como el amor, la amistad, el cariño, el apoyo mutuo, la identidad, la comunicación y el respeto.

Este pequeño proceso que se pudo observar en un período corto en donde se les ofreció a los jóvenes un tiempo para pensar y reflexionar, en donde no se les llenó de información, no se les ofrecían conclusiones, sino espacios para concluir. Por supuesto que hubo resistencias y dificultades para poder comunicarse, aún así sus respuestas fueron mucho más enriquecedoras.

Se puede observar, en parte, la presencia de una cultura del tener en ellos, no había información previa, eso les producía desconcierto, dificultad de verse a sí mismos y empezar a construirse como personas, con una opinión propia. Ingenuo sería pensar que en ese tiempo se logró dar una transferencia de la cultura del tener a la cultura del ser, es imposible, solo se les ofreció un tiempo para que lo pensaran. Algunos lograron interiorizar dentro de sí mismos nuevos valores, 
lograron autoafirmarse y tomar conciencia. Otros, simplemente se desacomodaron un poco y luego se acomodaron dentro de la "procesión de la vida".

Ese es parte del trabajo, no perder la dirección de la evolución, y tener mucha paciencia y fe de que poco a poco se dará el cambio, algunos ya lo iniciaron, otros lo "olieron" y otros ni siquiera lo notaron.

\section{Conclusiones}

El abuso sexual es apenas una manifestación más de una sexualidad del TENER, del placer, del egoísmo humano, en donde no existe el respeto, no hay edad, no hay vínculo y no hay libertad.

Saber que mi sexualidad forma parte de un proceso de evolución, no solo de reproducción o de maternidad y que debo tomar conciencia de que soy algo más que un delantal o una buena comida, es apenas el principio de un concepto y de un estilo de vida.

Descubrir quiénes somos, y lo profundamente extraordinario que es nuestro significado para la humanidad entera, hará que comencemos un período de respeto y mutua solidaridad para lograr que cada uno de nosotros SEA, y comience a formar parte de un nuevo cambio, que nos llevará otro tanto de siglos, necesarios en la reconstrucción de un cosmos que nos vió nacer mucho antes de que existiéramos.

Un espacio para ser, requiere de autoridad, no impuesta, autoridad revestida de conciencia, trascendencia y reflexión. Darle una dirección evolutiva a esta vida será el eje que nos mueva y no nos permita volver a la rutina de dejarnos morir en la alienación del no pensamiento y el no sentimiento.

Pero no es solo un proceso interior, es también un compromiso con los demás, con las personas que tengo a mi lado, con las que no tengo a mi lado, es dejar la individualidad, y la individualidad de hasta mi barrio, para poder trascender y abarcar a la humanidad por medio de uno solo. Así es como logro llegar a SER, como parte del todo, pero considerando a cada una de sus partes, sabiendo que existen, que dan aportes, que merecen respeto, que forman y dan sustento a la unidad (de la cual formamos parte todos y todas).

Una lucha que apenas inicia y que pierde su dimensión de lucha encarnizada para volverse en un proceso liberador de pensamiento y sentimiento.

En realidad, basado en lo anterior, los programas y talleres para la educación sexual debieran ser planeados cuidadosamente por cada comunidad para respetar la diversidad de los valores y de las creencias presentes en el salón de clase. 
Aunque los programas de educación sexual en las escuelas y colegios han estado en función por muchos años, estos no han sido efectivos como se esperaba. Es necesario que se realicen más estudios en donde se analice a profundidad lo que los jóvenes desean, lo que las comunidades esperan y lo que el Gobierno quiere con sus políticas de atención al adolescente.

\section{Referencias}

Cavanagh. (En Mezerville, Gastón) (1999). Madurez sacerdotal y religiosa. Tomos I y II. Bogotá: Consejo Episcopal Latinoamericano

Frankl, V. (1994). El hombre en busca de sentido. Madrid: Biblioteca de Psicología.

Fromm, E. (1992). ¿Ser o tener?. México: Fondo de Cultura Económica.

Fromm, E. (1992). Del tener al ser. Los recodos de la conciencia. México D.F.: Fondo de Cultura Económica.

Murillo, M. (1998). Los talleres de educación sexual con adolescentes. Ponencia, Congreso Nacional de Psicología. San José, Costa Rica.

Murillo, M. (Noviembre, 1999). Un encuentro conmigo. Taller. Foro de las Américas. Educación en valores. Tlaxcala, México.

Murillo, M. (Noviembre, 1999). Educación para una relación más profunda con la vida. Ponencia. Foro de las Américas. Tlaxcala.

Murillo, M. (2002). Lo cualitativo y lo cuantitativo. Una experiencia de campo. Metodología de abordaje en educación sexual. Congreso Nacional de Psicología, Costa Rica.

Murillo, M. (2001). La elaboración que un grupo de adolescentes de décimo nivel hacen acerca de la conceptualización de la sexualidad. Tesis para optar el grado de Maestría en Psicología. Sistema de Estudios de Posgrado. Maestría en Investigación en Psicología. Universidad de Costa Rica.

Murillo, M. (Abril, 2004). El lenguaje de la sexualidad. Propuestas interdisciplinarias en Educación Sexual. Congreso Nacional de Sexualidad. San José: Memorias del Congreso. 
Ross, M. P. (1998). Modelo Global de Intervención en Sexología Clínica. Formación y capacitación. San José: Asociación de Religiosos y Religiosas de Costa Rica.

Sanz, F. (1999). Psicoerotismo femenino y masculino. Para unas relaciones placenteras, autónomas y justas. Barcelona: Editorial Kairós. 